\title{
Preparation, Purity, and Homogeneity of NBS Standard Samples 705 and 706, Polystyrene
}

\author{
D. Mclntyre* \\ Institute for Materials Research, National Bureau of Standards, Washington, D.C. 20234
}

(September 14, 1966)

\begin{abstract}
The state-of-art in the measurement of the molecular weights of macromolecules is discussed. The preparation of polystyrene standard samples 705 and 706 is described. The results and the description of the carbon-hydrogen microanalysis, the ash determination, the lithium analysis, and the volatiles determination are given. The sampling methods for determining the homogeneity of 705 and 706 are presented. Some evidence of heterogeneity appeared to be present, particularly for lot 705 , but the experiment did not provide conclusive evidence of heterogeneity of the material.
\end{abstract}

Key Words: Ash content, elemental analysis, homogeneity, lithium content, molecular weights, polymer, preparation, purity, sampling, standard sample, volatile content.

\section{Introduction}

For some time there has been a need for standard samples of polymers beyond the usual scientist-toscientist exchange of interesting and/or well-characterized polymers. This need has been met by several undertakings in recent years. The Commission on Macromolecules of the International Union of Pure and Applied Chemistry in 1950 undertook the distribution of four samples of polystyrene with a request that recipient laboratories report their data on the characterization of the molecular weight. The polystyrene samples were fractions from two polymers obtained by the thermal polymerization of bulk styrene. One fraction was taken from a polymer formed at 15 percent conversion of the monomer; the other three fractions were from a polymer prepared by a bulk polymerization carried to a very high conversion. The characterization results, reported by H. P. Frank and H. Mark [1], ${ }^{1}$ are summarized in table 1 . The weightaverage molecular weights $\left(M_{w}\right)$ of the three fractions reported are approximately $1 \times 10^{5}, 3.5 \times 10^{5}$ and $10 \times 10^{5}$. The distribution of the molecular weights in the respective fractions are all relatively large; the $M_{w} / M_{n}$ ratio is approximately 1.4 , where $M_{n}$ is the number-average molecular weight. For all of the fractions the range of reported $M_{n}$ 's determined by osmotic pressure measurements in the same solvent is always greater than 30 percent of the mean value, comparing only the results from more than four laboratories using the same conditions of measurement. The $M_{w}$ 's, where there are more than four results under the same

*Present address: Department of Chemistry, The University of Akron, Akron, Ohio.

' Figures in brackets indicate the literature references at the end of this paper. conditions, give a range that is always greater than 20 percent of the mean value. The intrinsic viscosities show much less variance in general, although the results in methyl ethyl ketone (MEK) are often more scattered than those in toluene. The viscosities of the MEK solutions have an average range which is 14 percent of the mean, whereas the range in toluene is 9 percent. Some of the measurement problems were attributed to the large polydispersity of the polymers.

TABLE 1. Summary of the IUPAC molecular weight intercomparison [1]

\begin{tabular}{c|c|c|c|c|c}
\hline Fraction & Solvent & $\begin{array}{c}\text { No. of } \\
\text { reports }\end{array}$ & Average & Range & $\begin{array}{c}\text { Range as } \\
\text { a \% of } \\
\text { average }\end{array}$ \\
\hline
\end{tabular}

Osmotic pressure measurements: $M_{n}$

\begin{tabular}{c|c|c|c|c|c}
\hline & & & & & \\
I & Toluene & 6 & $1,000,000$ & $790,000-1,110,000$ & 32 \\
II & MEK & 4 & 235,000 & $199,000-280,000$ & 34 \\
III & MEK & 5 & $\mathbf{6 8 0 , 0 0 0}$ & $474,000-852,000$ & 55 \\
IV & MEK & 5 & 86,000 & $77,500-100,000$ & 38 \\
\hline
\end{tabular}

Viscosity measurements: $[\eta \mid \mathrm{ml} / \mathrm{g}$

\begin{tabular}{c|c|c|c|c|c}
\hline & & & & & \\
I & MEK & 6 & 103.5 & $90-111$ & 16 \\
I & Benzene & 4 & 221 & $211-230$ & 9 \\
I & Toluene & 13 & 204 & $174-216$ & 21 \\
II & Toluene & 4 & 104 & $102-106$ & 4 \\
II & MEK & 5 & 61.7 & $54-69$ & 23 \\
III & Toluene & 4 & 228 & $223-234$ & 5 \\
III & MEK & 5 & 115 & $110-122$ & 10 \\
IV & Toluene & 4 & 48 & $47-49.5$ & 5 \\
IV & MEK & 5 & 30.2 & $29-31.5$ & 8 \\
\hline
\end{tabular}

Light scattering measurements: $M_{u}$

\begin{tabular}{c|c|c|c|c|c}
\hline & & & & & \\
II & MEK & 5 & 328,000 & $288,000-360,000$ & 21 \\
III & MEK & 5 & 960,000 & $840,000-1,050,000$ & 22 \\
IV & MEK & 4 & 110,300 & $96,000-124,000$ & 25 \\
\hline
\end{tabular}


In 1956 Milkovich, Szwarc, and Levy [2] pointed out a new kinetic mechanism that could lead to narrowly dispersed molecular weights in polymers. McCormick, Brower, and Kin [3] scaled up the above laboratory preparation to produce $300-\mathrm{g}$ quantities of polystyrene. This group was later able to produce several pounds of narrowly dispersed polystyrene by a similar technique. On behalf of the Commission on Macromolecules of the IUPAC, H. Mark in 1960 undertook to distribute three new polystyrene samples of narrow molecular weight distribution prepared by the polymerization technique described in [3]. The $M_{w}$ 's of the samples to be intercompared were nominally described as $8.2 \times 10^{4}, 2,39 \times 10^{5}$, and 3.56 $\times 10^{6}$. The polydispersity $\left(M_{w} / M_{n}\right)$ was stated to be $1.05,1.08$, and 1.24. In all cases the polydispersity of the test samples is much less than in the previous intercomparison. The final results of the exchange program are not yet published, but the results of the characterization of the lowest two molecular weights have been reported to the Commission and are to be published [4]. In order to assess in a cursory fashion the development of the measurement techniques, selected data from one solvent or one wavelength are presented in table 2, The reported values for the viscosity, osmotic pressure, and light-scattering measurements are in much better agreement than in the previous intercomparison (table 1), although the range of the viscosity, $[\eta]$, and light-scattering measurements is not much different in the two intercomparisons. The standard deviation calculated for all of the reported results is smaller in the second IUPAC intercomparison. There is a marked improvement in the standard deviation of the osmotic pressure measurements; however, the results do indicate that the determination of these parameters is beset by considerable uncertainty even in laboratories specializing in macromolecular research.

TABLE 2. Preliminary results on second IUPAC molecular weight intercomparison [4]

\begin{tabular}{|c|c|c|c|c|c|}
\hline Sample & Property & $\begin{array}{l}\text { No. } \\
\text { results }\end{array}$ & Average & Range & $\begin{array}{c}\text { Range } \% \text { of } \\
\text { average }\end{array}$ \\
\hline$S_{102}$ & $\left\{\begin{array}{l}{[\eta], \text { toluene }} \\
M_{n} \\
M_{w}\end{array}\right.$ & $\begin{array}{r}14 \\
8 \\
8\end{array}$ & $\begin{array}{c}39 \mathrm{ml} / \mathrm{g} \\
78,000 \\
88,000\end{array}$ & $\begin{array}{c}38.3-40.5 \\
75-82.3 \times 10^{3} \\
79-114\end{array}$ & $\begin{array}{c}5 \\
9 \\
40\end{array}$ \\
\hline$S_{111}$ & $\left\{\begin{array}{l}{[\eta], \text { toluene }} \\
M_{u} \\
M_{u}\end{array}\right.$ & $\begin{array}{l}12 \\
8 \\
8\end{array}$ & $\begin{array}{l}78 \mathrm{ml} / \mathrm{g} \\
210,000 \\
233,000\end{array}$ & $\begin{array}{c}76.5-80.3 \\
196-225 \times 10^{3} \\
196-270\end{array}$ & $\begin{array}{c}5 \\
14 \\
32\end{array}$ \\
\hline
\end{tabular}

Early in 1963 the National Bureau of Standards issued two standard polystyrene samples to help standardize the measurement of certain macromolecular properties. Over the course of several years the NBS improved the capability of measuring the molecular weights of high polymers. With the cooperation of H. W. McCormick and his group at Dow Chemical Company in Midland, it became possible to obtain in 1961 a sufficient quantity of narrowly dispersed polystyrene to justify an effort to certify molecular measure- ments on a polymer sample that could be issued to all scientists. It was decided that two polystyrene samples would be certified and made available through the NBS Standard Sample program, one sample with a narrow molecular weight distribution, and the other with a broad molecular weight distribution. The first sample, being a new and laboratory scale preparation, would be distributed in limited quantities; the latter sample, being a pilot plant or commercial preparation, would be available in larger quantities.

Both samples were intended for instrument calibrations, but the narrow molecular weight distribution sample could eliminate certain experimental difficulties connected with the presence of appreciable amounts of low molecular weight polymer like the unavoidable diffusion of low molecular weight molecules across the semipermeable membrane during osmotic pressure measurements. The dispersion of molecular weights about the mean is sufficiently small so that most molecular properties are almost those of a monodisperse high polymer. In contrast, the polymer sample of broad molecular weight distribution would much more closely be related to commercially available polymers. The use of such samples would help control laboratory uses and also serve to define the properties of polydisperse materials.

The determination of molecular weights and molecular weight distribution can be roughly separated into three classes based on the existing experimental techniques and their limitations of measurement. Some of these limitations have been discussed earlier [5]. There is a low molecular weight range $\left(\sim 10^{4}\right)$, a moderate molecular weight range $(0.4-40) \times 10^{5}$, and a high molecular weight range $\left(>4 \times 10^{6}\right)$. The middle of the moderate range is experimentally very favorable, compared to the other ranges. In consideration of the known measurement techniques in 1958, the measurement program at the NBS was aimed at the improvement of technqiues to determine accurate molecular weights in the molecular weight range of 50,000 to 500,000 [5]. Later emphasis was to be given to the development of special techniques for $M_{n}$ determinations in the low molecular weight range. It was felt that though the high molecular weight range had tremendous importance for biological systems, it would be deferred until the low molecular weight range could be measured accurately. The commercial importance of both the low and moderate molecular weight range polymers implied that both broad and narrow molecular weight polymer standards should ultimately be available for both ranges of molecular weight.

The choice of the polymer to be issued as a standard is dictated by two simple questions: (1) Is there a polymer in the correct molecular weight range which can be supplied in a narrowly dispersed and also a broad dispersed sample, and (2) is this polymer reasonably stable and soluble in ordinarily used organic solvents? Polystyrene satisfies both of these requirements. A water-soluble polymer would have many additional advantages for those scientists interested in studying naturally occurring polymers. However many syn- 
thetic polymers, though not water soluble, are easily dissolved and stable in simple organic solvents. Polystyrene is not crystalline when it is derived from the usual free-radical or homogeneous anionic polymerization of styrene, yet it can be dissolved and handled in solution at high temperatures. Thus it can serve to standardize high temperature molecular weight measurements for crystalline polymers which are not soluble at lower temperatures as well. The unknown stereo-regularity of polystyrene is its only serious disadvantage for a macromolecular standard. Nevertheless, unknown stereo-regularity should not affect any molecular weight measurements, although fractionation efficiencies and transport properties could, to some small extent, be affected.

The results of the envisioned program to measure some of the molecular properties of a narrow and broad MWD polystyrene samples at moderate molecular weights will be presented in a series of papers. This first paper deals with the preparation, purity, and homogeneity of the samples referred to as NBS Polystyrene Standard Sample 705 and 706, respectively. Successive papers will discuss other aspects of the solution properties of these samples, such as the extinction coefficient, the partial specific volume, the osmotic pressures, turbidities, viscosities, and concentration gradients in a centrifugal field, measured at various initial concentrations in different solvents, as well as the phase diagrams and fractionation curves determined by separations from two coexisting liquid phases.

\section{Preparation of Samples}

\subsection{Polystyrene Standard Sample 705}

The styrene was polymerized in benzene solution using butyl lithium by McCormick and co-workers [3]. The polymer was precipitated from the benzene solution by adding methanol. The precipitate was then dried in a vacuum oven for 15 to $20 \mathrm{hr}$ at $75^{\circ} \mathrm{C}$. The fibrous precipitate was then densified by calendering momentarily at $175^{\circ} \mathrm{C}$. The polymer was then ground, injection molded at a high temperature, and cut into pellets weighing approximately $10 \mathrm{mg}$.

\subsection{Polystrene Standard Sample 706}

Pure styrene was thermally polymerized at $140^{\circ} \mathrm{C}$ to a conversion of 37.2 percent. The polymer was carefully heated and the residual monomer was pumped off at an elevated temperature. The polymer was then injection molded and cut into pellets weighing approximately $80 \mathrm{mg}$.

\section{Sampling of Polymers}

Polystrene 705 - The entire original sample, shipped in a carton, was placed in three amber jars. From each of these three jars, two 2 :g samples were removed, one about one-fourth the distance from the top of the jar, and the other about one-fourth the distance from the bottom of the jar. These six samples from six different locations constituted the samples for the study of the heterogeneity of the polymer, referred to later as heterogeneity samples. After the study of heterogeneity was concluded, two 50 -g representative samples of the entire supply of polymer were formed by emptying and rebottling the entire sample while retaining one scoop from every twenty scoops, each scoopful weighing $10 \mathrm{~g}$. These pooled and homogenized samples were called final samples. Measurements of the molecular properties were then made on these final samples.

Polystyreme 706 -Six 10-g samples were taken from the barrel of material to test the homogeneity of the sample. Each sample was taken from a different level of the barrel and at a different angular position with respect to the center of the barrel. The first sample was taken near the top center and the other samples from positions which formed a spiral path of constant descent. Two 500-g final samples were formed by collecting alternately $20 \mathrm{~g}$ of polymer from 250 -g scoopfuls, while emptying the barrel. These pooled samples were then thoroughly mixed and called final samples. The measurements of the molecular properties were made on these final samples.

\section{Heterogeneity of Samples}

Polystyrene 705 -Since the polymer standards were initially planned for molecular weight calibrations, the tests for homogeneity of the sample were based on the simplest measurements related to molecular weight, namely, the measurement of the intrinsic viscosities in a good solvent and the osmotic pressures in an ideal solvent. The percent of volatiles in the samples also was measured for samples taken from the top and the center of the barrel of Polystyrene 706; however, the differences in volatile content were not significant compared to the experimental error in the determination.

Cyclohexane at $35{ }^{\circ} \mathrm{C}$ was considered to be an ideal solvent for these samples, and the number-average molecular weight was therefore determined simply by the van't Hoff relation $\left(M_{n}=R T c / \pi\right)$ using a single, fixed concentration, where $\pi$ is the osmotic pressure, $c$ is the concentration, $T$ is the absolute temperature, and $R$ is the gas constant. The osmotic pressures of two independently made solutions of each heterogeneity sample were determined in cyclohexane at $35^{\circ} \mathrm{C}$. The osmotic pressure measurements were made with 600 gel cellophane in osmometers described in reference [7]. Previous work [8] had established the fact that the variation of ideality with molecular weight was very small, unless the end groups were quite different chemically from the backbone.

The intrinsic viscosities of the heterogeneity samples were determined in benzene at $25^{\circ} \mathrm{C}$ with an Ubbelohde viscometer having a flow time of approximately $200 \mathrm{sec}$, and a maximum shear rate of $\sim 1700$ 
$\sec ^{-1}$. The intrinsic viscosities were calculated by an extrapolation to zero concentration of the reduced specific viscosities for one initial concentration, $\sim 0.3$ $\mathrm{g} / \mathrm{dl}$, and two serial dilutions.

Table 3 reports the $M_{n}$ from osmotic pressure measurements and the intrinsic viscosities of the heterogeniety samples. The sample numbers, $(1,2,3)$, refer to the storage bottles and top (T) and bottom (B) to the location of the sample. The intrinsic viscosity of B-1 was determined three times because the average of the first two values was so much higher than the other averages, and an error in weighing was suspected. The third value also is high. An analysis of variance was performed to evaluate the variances within and between samples. For both the viscosity and the osmotic pressure measurements, the variability between different locations was found to be somewhat larger than that between duplicate measurements carried out on the same sample. However, the ratio of the between-to-within variability failed to reach significance at the 5-percent level. Thus, the experiment did not show conclusive evidence of heterogeneity of the material.

TABLE 3. Tests for homogeneity of polystyrene $705^{*}$

\begin{tabular}{c|c|c}
\hline \hline Sample & $M_{n}$ & $\begin{array}{c}{[\eta] .(\mathrm{ml} / \mathrm{g}) \text { benzene }} \\
\text { at } 25{ }^{\circ} \mathrm{C}\end{array}$ \\
\hline & 170,$000 ; 171,200$ & $75.1,74.2$ \\
T-1 & 170,$800 ; 170,800$ & $74.3,74.3$ \\
T-2 & 171,$300 ; 171,400$ & $74.6,74.3$ \\
T-3 & 172,$500 ; 171,900$ & $74.8,75.1,76.0$ \\
B-1 & 172,$200 ; 144,800$ & $74.4,74.0$ \\
B-2 & 172,$700 ; 175,600$ & $73.8,73.6$ \\
B-3 & \\
\hline
\end{tabular}

*These values do not represent the values listed on the certificate. The certificate value was determined from a larger number of determinations on a pooled sample.

Polystyrene 706-The intrinsic viscosities were measured in an Ubbelohde viscometer, having a flow time of $200 \mathrm{sec}$ for benzene and a maximum rate of shear of $1700 \mathrm{sec}^{-1}$, respectively. The intrinsic viscosities were determined by extrapolations of the reduced specific viscosity measured on a solution of polymer in benzene made by weight $(\sim 0.3 \mathrm{~g} / \mathrm{dl})$, and two other solutions made by serial dilutions of the original concentration in the viscometer. The intrinsic viscosities for different heterogeneity samples in benzene at $25{ }^{\circ} \mathrm{C}$ are reported in table 4 . An analysis of variance was performed and indicated no conclusive evidence of heterogeneity. This may be due to the fact that the error in the determination of the molecular weight by the simple intrinsic viscosity measurements is large enough to obscure any small heterogeneity in the lot of material. No further attempts at heterogeneity corrections were made. A number-average determination probably would have been revealing, but at the time of the measurements no suitable combination of membrane and osmometer was capable of measuring the osmotic pressure in a way that would give meaningful information on the low molecular weight species that are present.
TABLE 4. Test for homogeneity of polystyrene $706^{*}$

\begin{tabular}{c|c}
\hline \hline $\begin{array}{c}\text { Sample (in } \\
\text { sequence) }\end{array}$ & $\begin{array}{c}{[\eta],(\mathrm{ml} / \mathrm{g}) \text { benzene }} \\
\text { at } 25{ }^{\circ} \mathrm{C}\end{array}$ \\
\hline & \\
1 & $94.2,94.6$ \\
2 & $92.6,94.4$ \\
3 & $94.7,93.2$ \\
4 & $92.5,93.8$ \\
5 & $94.2,92.8$ \\
6 & $93.1,94.1$ \\
\hline
\end{tabular}

*These values do not represent the values listed on the certificate. The certificate value was determined from a larger number of determinations on a pooled sample.

\section{Chemical Analysis}

The styrene used in the polymerization of both 705 and 706 was commercial feedstock styrene. Fivemilligram portions of polystyrene from the final sample of both 705 and 706 were analyzed for carbon and hydrogen. The lithium content of sample 705 was determined on the ash by flame photometry. The values are close to the limit of detection. Table 5 lists the average experimental values and the assumed carbon and hydrogen percentages calculated from the formula $\mathrm{C}_{8} \mathrm{H}_{8}$. The total percent of carbon, hydrogen, and ash accounts for all of the material within experimental error. The carbon and hydrogen analyses are in good agreement with the formula $\mathrm{C}_{8} \mathrm{H}_{8}$. (The actual value in both cases is very close: 705 is $\mathrm{C}_{8} \mathrm{H}_{7.86}$ and 706 is $\mathrm{C}_{8} \mathrm{H}_{7.97 .}$.)

The ash content was independently determined by firing in a muffle, using a 1 -g and 2 -g sample of 705 , and a $2.5 \mathrm{~g}$ and $4.7-\mathrm{g}$ sample of 706 . Only 10 and $20 \mu \mathrm{g}$ of ash were found in the samples of 706 . The ash content is very low for 706 . The ash content of the 705 is surprisingly high, considering the care necessary to carry out such polymerizations. The lithium content of $\sim 0.005$ percent is approximately what is expected for a polymerization involving one lithium atom for every growing chain, i.e., 6.94/170,900 .004 percent.

TABLE 5. Chemical analyses of 705 and 706

\begin{tabular}{l|c|c|c}
\hline \hline \multirow{2}{*}{ \% Carbon } & Theoretical & 705 & 706 \\
\cline { 2 - 4 } & 92,257 & $92,2\left\{\begin{array}{l}92,07 \\
92,23 \\
92,22\end{array}\right.$ & $92,05\left\{\begin{array}{l}92,05 \\
92,05\end{array}\right.$ \\
\% Hydrogen & 7.743 & $7.7\left\{\begin{array}{l}7.75 \\
7.69 \\
7.74\end{array}\right.$ & $7.7\left\{\begin{array}{l}7.75 \\
7.63\end{array}\right.$ \\
\% Ash & & $0.036\left\{\begin{array}{l}0.034 \\
0.037\end{array}\right.$ & $<0.001$ \\
\% Lithium & & $0.005\left\{\begin{array}{l}0.004 \\
0.005\end{array}\right.$ & \\
Total & & 99.9 & 99.8 \\
\hline
\end{tabular}

\section{Volatiles}

The percent by weight of volatiles in the two standard samples was more difficult to determine than might 
be expected. Since both samples were prepared in their final form in the same manner, namely, devolatilization at high temperatures and reduced pressure, followed by extrusion, it is not surprising that both samples contain appreciable amounts of volatiles. For 705 the volatiles would primarily be benzene, for 706 the volatiles would be styrene. Both volatile contaminants are good solvents for the polymer and both have the same molecular size as the polymer repeating unit so that the diffusion of the last traces of the residual volatiles might be very difficult below the glass temperature of polystyrene $\left(90\right.$ to $\left.100{ }^{\circ} \mathrm{C}\right)$.

A drying apparatus had been constructed which allowed relatively large samples $(\sim 100 \mathrm{mg})$ to be dried under a high vacuum $\left(\sim 10^{-6} \mathrm{~mm} \mathrm{Hg}\right)$ for long times at various temperatures. One difficulty with any simple drying technique is the necessity of crushing the pellets in air to allow better diffusion of the trapped solvent out of the polymer into the cold trap. Table 6 reports the results of drying the polymer several times under different conditions of time and temperature. Neither the length of time (greater than $18 \mathrm{hr}$ ), nor the temperature (greater than $100^{\circ} \mathrm{C}$ ), made much difference in the weight loss. The average value is calculated for the weight loss at $100{ }^{\circ} \mathrm{C}$ over approximately $20 \mathrm{hr}$. If the crushing of 706 is continued vigorously for a long time, the weight loss is found to be less than 0.8 percent.

TABLE 6. Volatiles in 705 and 706 by weight loss measurements

\begin{tabular}{|c|c|c|c|}
\hline \multicolumn{4}{|c|}{ Sample 705} \\
\hline Run & $\%$ Loss & Time (hr) & Temp. ${ }^{\circ} \mathrm{C}$ \\
\hline $\begin{array}{ll}2 & \\
3 & (\text { Ave })^{*}\end{array}$ & $\begin{array}{c}0.49^{*} \\
.49 \\
.51 \\
.37^{*} \\
.69^{*} \\
0.52\end{array}$ & $\begin{array}{l}18 \\
42 \\
24 \\
24 \\
22\end{array}$ & $\begin{array}{l}100 \\
100 \\
115 \\
100 \\
100\end{array}$ \\
\hline \multicolumn{4}{|c|}{ Sample 706} \\
\hline $\begin{array}{lrr}2 & \\
3 & \\
4 & \\
& & \\
& & \\
\text { Ave })^{*}\end{array}$ & $\begin{array}{c}0.68^{*} \\
.67 \\
.73 \\
1.01^{*} \\
0.67^{*} \\
.91^{*} \\
0.82\end{array}$ & $\begin{array}{l}18 \\
42 \\
24 \\
24 \\
18 \\
18\end{array}$ & $\begin{array}{l}100 \\
100 \\
115 \\
100 \\
100 \\
100\end{array}$ \\
\hline
\end{tabular}

Samples of both 705 and 706 were heated to $100{ }^{\circ} \mathrm{C}$ in separate closed containers for $8 \mathrm{hr}$. The containers were then cooled in liquid nitrogen in order to pump off the residual air. The containers were then warmed to room temperature, and the volatilized material was analyzed mass spectrometrically. Under these conditions the weight loss was very small $(\sim 0 \%$ for 705 and $0.01 \%$ for 706). The analysis of the volatiles produced in the above treatment showed mainly water (92 mole percent in 705, 97 in 706) with traces of ben- zene in 705 ( 8 mole percent) and styrene in 706 ( 3 mole percent). The major contaminants, benzene and styrene, are those volatiles to be expected from the sample preparation, although the adsorbed water is the predominant species pumped off at room temperature. The determination of the residual styrene by a spectrophotometric analysis is discussed in a succeeding paper.

An analysis of the thermal degradation of both 705 and 706 appears in reference [9]. The weight loss in achieving the lowest temperatures reported for the kinetic studies $\left(\sim 350{ }^{\circ} \mathrm{C}\right)$ was about 0.5 percent for 705 and 1 percent for 706 .

\section{Packaging}

The packaging was carried out to avoid those pellets which had some gross physical imperfections, such as occluded foreign matter. Sample 705 is packaged in 2 -g lots in amber bottles to minimize degradation. Sample 706 is packaged in $20 \mathrm{~g}$ amounts. A small quantity has been stored in a freezer and will be compared in a few years with samples stored at room temperature.

The author thanks the following scientists both in and outside of the NBS, who have helped in this standards effort: H. McCormick and R. Boyer of the Dow Chemical Company, who arranged to make the polymers available to the NBS program; G. M. Kline and N. Bekkedahl for initiating the program; J. Mandel for the statistical analysis of some of the data; R. A. Paulson for the chemical analyses; and J. H. O'Mara, D. E. Roberts, J. McElwain, and D. Starr for many of the measurements and the detailed handling procedures.

\section{References}

[1] H. P. Frank and H. Mark, J. Polymer Sci. 10, 129 (1953).

[2] R. Milkovich, M. Szwarc, and M. Levy, J. Am. Chem. Soc., 78, 2656 (1956).

[3] H. W. McCormick, F. M. Brower, and L. Kin, J. Polymer Sci. 39, 87 (1959).

[4] Private communication, Dr. Mark.

[5] D. McIntyre, Unsolved Problems in Polymer Science, NASNRS Publication No. 995 (1962).

[6] Donald McIntyre, Symposium on Plastics Testing : and Standardization, ASTM Special Technical Publication No. 247 (1958).

[7] Donald McIntyre, J. Res. NBS 62, 63 (1959) (RP 2931).

[8] Donald McIntyre, J. H. O'Mara, and B. C. Konouck, J. Am. Chem. Soc. 81, 3498 (1959).

[9] L. A. Wall, S. Straus, J. H. Flynn, D. McIntyre, and R. Simha, J. Phys. Chem. 70, 53 (1966).

(Paper 71A1-435) 